\title{
Integral Estimates for the Potential Operator on Differential Forms
}

\author{
Casey Johnson and Shusen Ding \\ Department of Mathematics, Seattle University, Seattle, WA 98122, USA \\ Correspondence should be addressed to Shusen Ding; sding@seattleu.edu
}

Received 2 October 2012; Accepted 21 November 2012

Academic Editor: Tohru Ozawa

Copyright (C) 2013 C. Johnson and S. Ding. This is an open access article distributed under the Creative Commons Attribution License, which permits unrestricted use, distribution, and reproduction in any medium, provided the original work is properly cited.

We develop the local inequalities with new weights for the potential operator applied to differential forms. We also prove the global weighted norm inequalities for the potential operator in averaging domains and explore applications of our new results.

\section{Introduction}

This paper deals with the weighted estimates for the potential operator applied to differential forms. Throughout this paper, $\Omega$ will denote an open subset of $\mathbb{R}^{n}, n \geq 2$, and $\mathbb{R}=\mathbb{R}^{1}$. Let $e_{1}=(1,0, \ldots, 0), e_{2}=(0,1, \ldots, 0), \ldots, e_{n}=(0,0, \ldots, 1)$ be the standard unit basis of $\mathbb{R}^{n}$. For $l=0,1, \ldots, n$, the linear space of $l$-vectors, spanned by the exterior products $e_{I}=e_{i_{1}} \wedge e_{i_{2}} \wedge$ $\cdots \wedge e_{i_{l}}$, corresponding to all ordered $l$-tuples $I=\left(i_{1}, i_{2}, \ldots, i_{l}\right)$, $1 \leq i_{1}<i_{2}<\cdots<i_{l} \leq n$, is denoted by $\wedge^{l}=\wedge^{l}\left(\mathbb{R}^{n}\right)$. The Grassman algebra

$$
\wedge=\wedge\left(\mathbb{R}^{n}\right)=\stackrel{n}{\oplus} \wedge\left(\mathbb{R}^{n}\right)
$$

is a graded algebra with respect to the exterior products. For $\alpha=\sum \alpha^{I} e_{I} \in \wedge$ and $\beta=\sum \beta^{I} e_{I} \in \wedge$, the inner product in $\wedge$ is given by

$$
\langle\alpha, \beta\rangle=\sum \alpha^{I} \beta^{I}
$$

with summation over all $l$-tuples $I=\left(i_{1}, i_{2}, \ldots, i_{l}\right)$ and all integers $l=0,1, \ldots, n$. We should also notice that $d x_{i} \wedge d x_{j}=$ $-d x_{j} \wedge d x_{i}, \quad i \neq j$, and $d x_{i} \wedge d x_{i}=0$.

Assume that $B \subset \mathbb{R}^{n}$ is a ball and $\sigma B$ is the ball with the same center as $B$ and with diam $(\sigma B)=\sigma \operatorname{diam}(B)$. Differential forms are extensions of functions defined in
$\mathbb{R}^{n}$. A function $f\left(x_{1}, \ldots, x_{n}\right)$ in $\mathbb{R}^{n}$ is called a 0 -form. A differential $l$-form is of the form

$$
\begin{aligned}
w(x) & =\sum_{I} w_{I}(x) d x_{I} \\
& =\sum w_{i_{1} i_{2} \cdots i_{l}}(x) d x_{i_{1}} \wedge d x_{i_{2}} \wedge \cdots \wedge d x_{i_{l}}
\end{aligned}
$$

in $\mathbb{R}^{n}$. Differential forms have become invaluable tools for many fields of sciences and engineering, including theoretical physics, general relativity, potential theory, and electromagnetism. They can be used to describe various systems of PDEs and to express different geometrical structures on manifolds. Many interesting and useful results about the differential forms have been obtained during recent years; particularly, for the differential forms satisfying some version of $A$ hrmonic equation, see [1-8]. The $n$-dimensional Lebesgue measure of a set $E \subseteq \mathbb{R}^{n}$ is denoted by $|E|$. We call $w$ a weight if $w \in L_{\text {loc }}^{1}\left(\mathbb{R}^{n}\right)$ and $w>0$ a.e. For $0<p<\infty$, we denote the weighted $L^{p}$ norm of a measurable function $f$ over $E$ by

$$
\|f\|_{p, E, w^{\alpha}}=\left(\int_{E}|f(x)|^{p} w^{\alpha} d x\right)^{1 / p}
$$

if the above integral exists. Here $\alpha$ is a real number. It should be noticed that the Hodge star operator can be defined equivalently as follows. 
Definition 1. If $\omega=\alpha_{i_{1}, i_{2}, \ldots, i_{k}}\left(x_{1}, x_{2}, \ldots, x_{n}\right) d x_{i_{1}} \wedge d x_{i_{2}} \wedge \cdots \wedge$ $d x_{i_{k}}=\alpha_{I} d x_{I}, i_{1}<i_{2}<\cdots<i_{k}$, is a differential $k$-form, then

$$
\star \omega=\star \alpha_{i_{1} i_{2} \cdots i_{k}} d x_{i_{1}} \wedge d x_{i_{2}} \wedge \cdots \wedge d x_{i_{k}}=(-1)^{\sum(I)} \alpha_{I} d x_{J},
$$

where $I=\left(i_{1}, i_{2}, \ldots, i_{k}\right), J=\{1,2, \ldots, n\}-I$, and

$$
\sum(I)=\frac{k(k+1)}{2}+\sum_{j=1}^{k} i_{j}
$$
[8].

The following $A(\alpha, \beta, \gamma ; E)$-weights were introduced in

Definition 2. One says that a measurable function $g(x)$ defined on a subset $E \subset \mathbb{R}^{n}$ satisfies the $A(\alpha, \beta, \gamma ; E)$ condition for some positive constants $\alpha, \beta, \gamma$, writes $g(x) \in$ $A(\alpha, \beta, \gamma ; E)$ if $g(x)>0$ a.e., and writes

$$
\sup _{B}\left(\frac{1}{|B|} \int_{B} g^{\alpha} d x\right)\left(\frac{1}{|B|} \int_{B} g^{-\beta} d x\right)^{\gamma / \beta}<\infty,
$$

where the supremum is over all balls $B \subset E$. One says that $g(x)$ satisfies the $A(\alpha, \beta ; E)$-condition if (7) holds for $\gamma=1$ and write $g(x) \in A(\alpha, \beta ; E)=A(\alpha, \beta, 1 ; E)$.

Notice that there are three parameters in the definition of the $A(\alpha, \beta, \gamma ; E)$-class. We obtain some existing weighted classes if we choose some particular values for these parameters. For example, it is easy to see that the $A(\alpha, \beta, \gamma ; E)$-class reduces to the usual $A_{r}(E)$-class if $\alpha=\gamma=1$ and $\beta=1 /(r-1)$.

Recently, Bi extended the definition of the potential operator to the case of differential forms; see [2]. For any differential $k$-form $w(x)$, the potential operator $P$ is defined by

$$
P w(x)=\sum_{I} \int_{E} K(x, y) w_{I}(y) d y d x_{I}
$$

where the kernel $K(x, y)$ is a nonnegative measurable function defined for $x \neq y$ and the summation is over all ordered $k$-tuples $I$. The $k=0$ case reduces to the usual potential operator,

$$
P f(x)=\int_{E} K(x, y) f(y) d y,
$$

where $f(x)$ is a function defined on $E \subset \mathbb{R}^{n}$. A kernel $K$ on $\mathbb{R}^{n} \times \mathbb{R}^{n}$ is said to satisfy the standard estimates if there are $\delta, 0<\delta \leq 1$, and constant $C$ such that, for all distinct points $x$ and $y$ in $\mathbb{R}^{n}$ and all $z$ with $|x-z|<(1 / 2)|x-y|$, the kernel $K$ satisfies (i) $K(x, y) \leq C|x-y|^{-n}$, (ii) $\mid K(x, y)-$ $K(z, y)|\leq C| x-\left.z\right|^{\delta}|x-y|^{-n-\delta}$, and (iii) $|K(y, x)-K(y, z)| \leq$ $C|x-z|^{\delta}|x-y|^{-n-\delta}$. In this paper, we always assume that $P$ is the potential operator with the kernel $K(x, y)$ satisfying the above condition (i) in the standard estimates. In [2], Bi proved the following inequality for the potential operator:

$$
\left\|P(u)-(P(u))_{E}\right\|_{p, E} \leq C|E| \operatorname{diam}(E)\|u\|_{p, E},
$$

where $u \in D^{\prime}\left(E, \wedge^{k}\right), k=0,1, \ldots, n-1$, is a differential form defined in a bounded and convex domain $E$.
Definition 3. One says that a differential form $u \in \Lambda^{l}(E)$ belongs to the $\operatorname{WRH}\left(\wedge^{l}, E\right)$-class and writes $u \in \operatorname{WRH}\left(\wedge^{l}, E\right)$, $l=0,1,2, \ldots, n$, if for any constants $0<s, t<\infty$, the inequality

$$
\|u\|_{s, B} \leq C|B|^{(t-s) / s t}\|u\|_{t, \sigma B}
$$

holds for any ball $B$ with $\sigma B \subset \Omega$, where $\sigma>1$ and $C>0$ are constants.

From [1], we know that any solution of $A$-harmonic equations satisfies (11). Hence, the $\operatorname{WRH}\left(\wedge^{l}, \Omega\right)$-class is a large class of differential forms. We will use the following Hölder inequality repeatedly in this paper.

Lemma 4. Let both $f$ and $g$ be measurable functions in $\mathbb{R}^{n}$ and $p, q>0$ and $1 / s=(1 / p)+(1 / q)$. Then

$$
\left(\int_{E}|f g|^{s} d x\right)^{1 / s} \leq\left(\int_{E}|f|^{p} d x\right)^{1 / p}\left(\int_{E}|g|^{q} d x\right)^{1 / q}
$$

for any $E \subset \mathbb{R}^{n}$.

\section{Local Inequalities}

In this section, we will prove some local weighted norm inequalities for the potential operator.

Theorem 5. Let $P$ be the potential operator applied to a differential form $u \in \mathrm{WRH}\left(\wedge^{l}, \Omega\right)$, where $\Omega \subset \mathbb{R}^{n}$ is a domain. Assume that $w(x) \in A(\alpha, \beta ; \Omega)$ with $\alpha, \beta>0$. Then, there exists a constant $C$, independent of $u$, such that

$$
\left\|P(u)-(P(u))_{B}\right\|_{s, B, w} \leq C|B| \operatorname{diam}(B)\|u\|_{s, B, w}
$$

for all balls $B$ with $B \subset \Omega$, where $s>1$ is a constant.

Proof. Let $t=s \alpha /(\alpha-1)$, then $t>s$. Using Lemma 4 with $1 / s=(1 / t)+((t-s) / s t)$ yields

$$
\begin{aligned}
\| P(u)- & (P(u))_{B} \|_{s, b, w} \\
= & \left(\int_{B}\left|P(u)-(P(u))_{B}\right|^{s} w d x\right)^{1 / s} \\
= & \left(\int_{B}\left(\mid P(u)-\left(\left.P(u)\right|_{B} w^{1 / s}\right)^{s} d x\right)^{1 / s}\right. \\
\leq & \left(\int_{B}\left|P(u)-(P(u))_{B}\right|^{t} d x\right)^{1 / t} \\
& \times\left(\int_{B}\left(w^{1 / s}\right)^{s t /(t-s)} d x\right)^{(t-s) / s t} \\
\leq & C_{1}\|B\| \operatorname{diam}(B)\|u\|_{t, B}\left(\int_{B} w^{\alpha}(x) d x\right)^{1 / \alpha s} .
\end{aligned}
$$

Set $k=\beta s /(1+\beta)$, then $0<k<s$. Since $u$ is in the $\operatorname{WRH}(\Omega)$ class,

$$
\|u\|_{t, B} \leq C_{2}\|B\|^{(k-t) / k t}\|u\|_{k, \sigma_{1} B}
$$


where $\sigma_{1}>1$ in a constraint. Since $u$ is in the $\mathrm{WRH}(\Omega)$-class again (note that $1 / k=(1 / s)+(s-k) / s k)$,

$$
\begin{aligned}
\|u\|_{k, \sigma_{1} B}= & \left(\int_{\sigma_{1} B}\left(|u| w^{1 / s} s^{-1 / s}\right)^{k} d x\right)^{1 / k} \\
\leq & \left(\int_{\sigma_{1} B}\left(|u| w^{1 / s} w^{-1 / s}\right)^{k} d x\right)^{1 / k} \\
& \times\left(\int_{\sigma_{1} B}\left(w^{-1 / s}\right)^{k s /(s-k)} d x\right)^{(s-k) / k s} \\
= & \left(\int_{\sigma_{1} B}|u|^{s} w d x\right)^{1 / s} \\
& \times\left(\int_{\sigma_{1} B} w^{-k /(s-k)} d x\right)^{(s-k) / k s} \\
= & \left(\int_{\sigma_{1} B}|u|^{s} w d x\right)^{1 / s}\left(\int_{\sigma_{1} B} w^{-\beta} d x\right)^{1 / \beta s},
\end{aligned}
$$

where we have used the following calculation:

$$
\begin{aligned}
\frac{k}{s-k} & =\frac{\beta s /(1+\beta)}{s-(\beta s /(1+\beta))}=\frac{\beta /(1+\beta)}{a-(\beta /(1+\beta))} \\
& =\frac{\beta /(1+\beta)}{(1+\beta-\beta) /(1+\beta)}=\beta .
\end{aligned}
$$

Combining (14), (15) and, (16) gives

$$
\begin{aligned}
\| P(u)- & (P(u))_{B} \|_{s, B, w} \\
\leq & C_{3}|B| \operatorname{diam}(B)|B|^{(k-t) / k t}\left(\int_{\sigma_{1} B}|u|^{s} w d x\right)^{1 / s} \\
& \times\left(\int_{B} w^{\alpha} d x\right)^{1 / \alpha s}\left(\int_{\sigma_{1} B} w^{-\beta} d x\right)^{1 / \beta s} .
\end{aligned}
$$

Note that since $w \in A(\alpha, \beta, \alpha ; \Omega)$, it follows that

$$
\begin{aligned}
& \left(\int_{B} w^{\alpha} d x\right)^{1 / \alpha s}\left(\int_{\sigma_{1} B} w^{-\beta} d x\right)^{1 / \beta s} \\
& =\left(\left(\int_{\sigma_{1} B} w^{\alpha} d x\right)\left(\int_{\sigma_{1} B} w^{-\beta} d x\right)^{\alpha / \beta}\right)^{1 / \alpha s} \\
& =\left(\left|\sigma_{1} B\right|^{1+(\alpha / \beta)}\left(\frac{1}{|\beta|} \int_{\sigma_{1} B} w^{\alpha} d x\right)\left(\frac{1}{|\beta|} \int_{\sigma_{1} B} w^{-\beta} d x\right)^{\alpha / \beta}\right)^{1 / \alpha s} \\
& =C_{4}|B|^{(1 / \alpha s)+(1 / \beta s)} .
\end{aligned}
$$

Plugging (19) into (18), we have

$$
\begin{aligned}
\| P(U)- & (P(u))_{B} \|_{s, B, w} \\
\leq & C_{5} \operatorname{diam}(B)|B|^{1+(1 / t)-(1 / k)} \\
& \times\left(\int_{\sigma_{1} B}|u|^{s} w d x\right)^{1 / s} C_{4}|B|^{(1 / \alpha s)+(1 / \beta s)} \\
\leq & C_{6} \operatorname{diam}(B)|B|^{1+(1 / t)-(1 / k)+(1 / \alpha s)+(1 / \beta s)} \\
& \times\left(\int_{\sigma_{1} B}|u|^{s} w d x\right)^{1 / s} .
\end{aligned}
$$

We should notice that

$$
1+\frac{1}{t}-\frac{1}{k}+\frac{1}{\alpha s}+\frac{1}{\beta s}=1+\frac{\alpha-1}{s \alpha}-\frac{1+\beta}{s \beta}+\frac{1}{\alpha s}+\frac{1}{\beta s}=1 .
$$

Combining (20) and (21) gives us

$$
\left\|P(u)-(P(u))_{B}\right\|_{s, B, w} \leq C|B| \operatorname{diam}(B)\left(\int_{\sigma_{1} B}|u|^{s} w d x\right)^{1 / s} .
$$

The proof of Theorem 5 has been completed.

A continuously increasing function $\varphi:[0, \infty) \rightarrow[0, \infty)$ with $\varphi(0)=0$ is called an Orlicz function. A convex Orlicz function $\varphi$ is often called a Young function. The Orlicz space $L^{\varphi}(\Omega, \mu)$ consists of all measurable functions $f$ on $\Omega$ such that $\int_{\Omega} \varphi(|f| / \lambda) d \mu<\infty$ for some $\lambda=\lambda(f)>0$. If $\varphi$ is a Young function, then

$$
\|f\|_{\varphi(\Omega, \mu)}=\inf \left\{\lambda>0: \int_{\Omega} \varphi\left(\frac{|f|}{\lambda}\right) d \mu \leq 1\right\}
$$

defines a norm in $L^{\varphi}(\Omega, \mu)$, which is called the Orlicz norm or the Luxemburg norm.

Definition 6 (see [9]). One says that a Young function $\varphi$ lies in the class $G(p, q, C), 1 \leq p<q<\infty, C \geq 1$, if (i) $1 / C \leq$ $\varphi\left(t^{1 / p}\right) / j(t) \leq C$ and (ii) $1 / C \leq \varphi\left(t^{1 / q}\right) / h(t) \leq C$ for all $t>0$, where $j$ is a convex increasing function and $h$ is a concave increasing function on $[0, \infty)$.

From [9], each of $\varphi, j$, and $h$ in the above definition is doubling in the sense that its values at $t$ and $2 t$ are uniformly comparable for all $t>0$ and the consequent fact that

$$
C_{1} t^{q} \leq h^{-1}(\varphi(t)) \leq C_{2} t^{q}, \quad C_{1} t^{p} \leq j^{-1}(\varphi(t)) \leq C_{2} t^{p},
$$

where $C_{1}$ and $C_{2}$ are constants. Also, for all $1 \leq p_{1}<$ $p<p_{2}$ and $\alpha \in \mathbb{R}$, the function $\varphi(t)=t^{p} \log _{+}^{\alpha} t$ belongs to $G\left(p_{1}, p_{2}, C\right)$ for some constant $C=C\left(p, \alpha, p_{1}, p_{2}\right)$. Here $\log _{+}(t)$ is defined by $\log _{+}(t)=1$ for $t \leq e$ and $\log _{+}(t)=\log (t)$ for $t>e$. Particularly, if $\alpha=0$, we see that $\varphi(t)=t^{p}$ lies in $G\left(p_{1}, p_{2}, C\right), 1 \leq p_{1}<p<p_{2}$. 
Theorem 7. Let $P$ be the potential operator applied to a differential form $u \in \mathrm{WRH}\left(\wedge^{l}, \Omega\right)$ and $\varphi$ a Young function in the class $G(p, q, C), 1 \leq p<q<\infty, C \leq 1$, and where $\Omega$ is a bounded domain. Assume that $\varphi(|u|) \in L_{\mathrm{loc}}^{1}(\Omega, \mu)$. Then, there exists a constant $M>0$, independent of $u$, such that

$$
\int_{B} \varphi\left(\frac{\left|P(u)-(P(u))_{B}\right|}{k}\right) d x \leq M \int_{\sigma B} \varphi\left(\frac{|u|}{k}\right) d x
$$

for all balls $B$ with $B \subset \Omega$, where $\sigma>1$ is a constant.

Proof. Using Jensen's inequality for $h^{-1}$ and (24), we have

$$
\begin{aligned}
\int_{B} \varphi & \left(\frac{\left|P(u)-(P(u))_{B}\right|}{k}\right) d x \\
& =h\left(h^{-1}\left(\int_{B} \varphi\left(\frac{\left|P(u)-(P(u))_{B}\right|}{k}\right) d x\right)\right) \\
& \leq h\left(\int_{B} h^{-1}\left(\varphi\left(\frac{\left|P(u)-(P(u))_{B}\right|}{k}\right)\right) d x\right) \\
& \leq h\left(C_{1} \int_{B}\left(\frac{\left|P(u)-(P(u))_{B}\right|}{k}\right)^{q} d x\right) \\
& \leq C_{2} \varphi\left(\left(C_{1} \int_{B}\left(\frac{\left|P(u)-(P(u))_{B}\right|}{k}\right)^{q} d x\right)^{1 / q}\right) \\
& \leq C_{2} \varphi\left(\frac{1}{k}\left(C_{1} \int_{B}\left(\left|P(u)-(P(u))_{B}\right|\right)^{q} d x\right)^{1 / q}\right) \\
& \leq C_{3} \varphi\left(\frac{1}{k}\left(\int_{B}\left(\left|P(u)-(P(u))_{B}\right|\right)^{q} d x\right)^{1 / q}\right) .
\end{aligned}
$$

Since $u \in \operatorname{WRH}(\Omega)$, by (11) we obtain

$$
\left(\int_{B}|u|^{q} d x\right)^{1 / q} \leq C_{4}|B|^{(p-q) / p q}\left(\int_{\sigma B}|u|^{p} d x\right)^{1 / p},
$$

where $\sigma>1$ is a constant. Using (13), (24), and Jensen's inequality,

$$
\begin{aligned}
& \varphi\left(\frac{1}{k}\left(\int_{B}\left|P(u)-(P(u))_{B}\right|^{q} d x\right)^{1 / q}\right) \\
& \quad \leq \varphi\left(\frac{1}{k}|B|^{1+(1 / n)}\left(\int_{B}|u|^{q} d x\right)^{1 / q}\right) \\
& \quad \leq \varphi\left(\frac{1}{k}|B|^{1+(1 / n)} C_{4}|B|^{(1 / q)-(1 / p)}\left(\int_{\sigma B}|u|^{p} d x\right)^{1 / p}\right)
\end{aligned}
$$

$$
\begin{aligned}
& =\varphi\left(\left(\frac{1}{k^{p}} C_{4}^{p}|B|^{p(1+(1 / n)+(1 / q)-(1 / p))} \int_{\sigma B}|u|^{p} d x\right)^{1 / p}\right) \\
& \leq C_{5} j\left(\frac{1}{k^{p}} C_{4}^{p}|B|^{p(1+(1 / n)+(1 / q)-(1 / p))} \int_{\sigma B}|u|^{p} d x\right) \\
& \leq C_{5} j\left(\int_{\sigma B} \frac{1}{k^{p}} C_{4}^{p}|B|^{p(1+(1 / n)+(1 / q)-(1 / p))}|u|^{p} d x\right) \\
& \leq C_{5} \int_{\sigma B} j\left(\frac{1}{k^{p}} C_{4}^{p}|B|^{p(1+(1 / n)+(1 / q)-(1 / p))}|u|^{p}\right) d x .
\end{aligned}
$$

Note that $p \geq 1$, then $1+(1 / n)+(1 / q)-(1 / p)>0$. Thus,

$$
|B|^{1+(1 / n)+(1 / q)-(1 / p)} \leq|\Omega|^{1+(1 / n)+(1 / q)-(1 / p)} \leq C_{6} .
$$

Using the above inequality and (i) in Definition 6, we find that $j(t) \leq C_{7} \varphi\left(t^{1 / p}\right)$. Therefore,

$$
\begin{aligned}
\int_{\sigma B} j & \left(\frac{1}{k^{p}} C_{4}^{p}|B|^{p(1+(1 / n)+(1 / q)-(1 / p))}|u|^{p}\right) d x \\
& \leq C_{7} \int_{\sigma B} \varphi\left(\frac{1}{k} C_{4}|B|^{1+(1 / n)+(1 / q)-(1 / p)}|u|\right) d x \\
& \leq C_{7} \int_{\sigma B} \varphi\left(C_{8} \frac{|u|}{k}\right) d x \\
& \leq C_{9} \int_{\sigma B} \varphi\left(\frac{|u|}{k}\right) d x .
\end{aligned}
$$

Combining (28) and (30) yields

$$
\varphi\left(\frac{1}{k}\left(\int_{B}\left|P(u)-(P(u))_{B}\right|^{q} d x\right)^{1 / q}\right) \leq C_{10} \int_{\sigma B} \varphi\left(\frac{|u|}{k}\right) d x .
$$

Finally, substituting (31) into (26), we obtain

$$
\int_{B} \varphi\left(\frac{\left|P(u)-(P(u))_{B}\right|}{k}\right) d x \leq M \int_{\sigma B} \varphi\left(\frac{|u|}{k}\right) d x .
$$

The proof of Theorem 7 has been completed.

\section{Global Inequalities}

In 1989, Staples introduced the following $L^{s}$-averaging domains in [10]. A proper subdomain $\Omega \subset \mathbb{R}^{n}$ is called an $L^{s}$-averaging domain, $s \geq 1$, if there exists a constant $C$ such that

$$
\left(\frac{1}{|\Omega|} \int_{\Omega}\left|u-u_{\Omega}\right|^{s} d x\right)^{1 / s} \leq C \sup _{B \subset \Omega}\left(\frac{1}{|\Omega|} \int_{B}\left|u-u_{B}\right|^{s} d x\right)^{1 / s}
$$

for all $u \in L_{\text {loc }}^{s}(\Omega)$. Here the supremum is over all balls $B \subset \Omega$. The $L^{s}$-averaging domains were extended into the $L^{s}(\mu)$-averaging domains recently in [11]. We call a proper 
subdomain $\Omega \subset \mathbb{R}^{n}$ an $L^{s}(\mu)$-averaging domain, $s \geq 1$, if $\mu(\Omega)<\infty$ and there exists a constant $C$ such that

$$
\begin{aligned}
& \left(\frac{1}{\mu(\Omega)} \int_{\Omega}\left|u-u_{B_{0}}\right|^{s} d \mu\right)^{1 / s} \\
& \quad \leq C \sup _{4 B \subset \Omega}\left(\frac{1}{\mu(B)} \int_{B}\left|u-u_{B}\right|^{s} d \mu\right)^{1 / s}
\end{aligned}
$$

for some ball $B_{0} \subset \Omega$ and all $u \in L_{\text {loc }}^{s}(\Omega ; \mu)$. The $L^{s}(\mu)$ averaging domain was genralized into the following $L^{\varphi}(\mu)$ averaging domain in [12].

Definition 8. Let $\varphi$ be a continuous increasing convex function on $[0, \infty)$ with $\varphi(0)=0$. One calls a proper subdomain $\Omega \subset \mathbb{R}^{n}$ an $L^{\varphi}(\mu)$-averaging domain, if $\mu(\Omega)<\infty$ and there exists a constant $C$ such that

$$
\begin{aligned}
& \frac{1}{\mu(\Omega)} \int_{\Omega} \varphi\left(\tau\left|u-u_{B_{0}}\right|\right) d \mu \\
& \quad \leq C \sup _{4 B \subset \Omega} \frac{1}{\mu(B)} \int_{B} \varphi\left(\sigma\left|u-u_{B}\right|\right) d \mu
\end{aligned}
$$

for some ball $B_{0} \subset \Omega$ and all $u$ such that $\varphi(|u|) \in L_{l o c}^{1}(\Omega ; \mu)$, where the measure $\mu$ is defined by $d \mu=w(x) d x, w(x)$ is a weight, and $\tau, \sigma$ are constants with $0<\tau<1,0<\sigma<1$, and the supremum is over all balls $B \subset \Omega$ with $4 B \subset \Omega$.

From the above definition, we see that $L^{s}(\mu)$-averaging domains are special $L^{\varphi}(\mu)$-averaging domains when $\varphi(t)=t^{s}$ in Definition 8.

Theorem 9. Let $\varphi$ be a Young function in the class $G(p, q, C)$, $1 \leq p<q<\infty, C \geq 1$; and $\Omega \subset \mathbb{R}^{n}$ any bounded $L^{\varphi}(\mu)$ averaging domain, and $P$ the potential operator applied to a differential form $u \in \mathrm{WRH}\left(\wedge^{l}, \Omega\right), l=1,2 \ldots, n$. Assume that $\varphi(|u|) \in L^{1}(\Omega, \mu)$. Then, there exists a constant $C$, independent of $u$, such that

$$
\int_{\Omega} \varphi\left(\frac{1}{k}\left|P(u)-(P(u))_{B_{0}}\right|\right) d \mu \leq C \int_{\Omega} \varphi\left(\frac{|u|}{k}\right) d \mu,
$$

where $B_{0} \subset \Omega$ is some fixed ball.

Proof. From Definition 8, (25), and noticing that $\varphi$ is doubling, we have

$$
\begin{aligned}
\int_{\Omega} \varphi & \left(\left|P(u)-(P(u))_{B_{0}}\right|\right) d \mu \\
& \leq C_{1} \sup _{B \subset \Omega} \int_{B} \varphi\left(\left|P(u)-(P(u))_{B}\right|\right) d \mu \\
& \leq C_{1} \sup _{B \subset \Omega}\left(C_{2} \int_{\sigma B} \varphi(|u|) d \mu\right) \\
& \leq C_{1} \sup _{B \subset \Omega}\left(C_{2} \int_{\Omega} \varphi(|u|) d \mu\right) \\
& \leq C_{3} \int_{\Omega} \varphi(|u|) d \mu .
\end{aligned}
$$

We have completed the proof of Theorem 9.
Choosing $\varphi(t)=t^{s} \log _{+}^{\alpha} t$ in Theorem 9, we obtain the following Poincaré inequalities with the $L^{s}\left(\log _{+}^{\alpha} L\right)$-norms.

Corollary 10. Let $\varphi(t)=t^{s} \log _{+}^{\alpha} t, s \geq 1, \alpha \in \mathbb{R}$, and $P$ the potential operator applied to a differential form $u \in$ $\mathrm{WRH}\left(\wedge^{l}, \Omega\right), l=1,2 \ldots, n$. Assume that $\varphi(|u|) \in L^{1}(\Omega, \mu)$. Then, there exists a constant $C$, independent of $u$, such that

$$
\begin{gathered}
\int_{\Omega}\left|P(u)-(P(u))_{B_{0}}\right|^{s} \log _{+}^{\alpha}\left(\left|P(u)-(P(u))_{B_{0}}\right|\right) d \mu \\
\leq C \int_{\Omega}|u|^{s} \log _{+}^{\alpha}(|u|) d \mu
\end{gathered}
$$

for any bounded $L^{\varphi}(\mu)$-averaging domain $\Omega$ and $B_{0} \subset \Omega$ is some fixed ball.

Note that (38) can be written as the following version with the Luxemburg norm:

$$
\left\|P(u)-(P(u))_{B_{0}}\right\|_{L^{s}\left(\log _{+}^{\alpha} L\right)(\Omega)} \leq C\|u\|_{L^{s}\left(\log _{+}^{\alpha} L\right)(\Omega)}
$$

provided that the conditions in Corollary 10 are satisfied.

\section{Applications}

We have established the local and global weighted estimates for the potential operator applied to the differential forms in the $\mathrm{WRH}\left(\wedge^{l}, \Omega\right)$-class. It is well known that any solution to $A$-harmonic equations belongs to the $\operatorname{WRH}\left(\wedge^{l}, \Omega\right)$-class. Hence, our inequalities can be used to estimate solutions of $A$-harmonic equations. Next, as applications of the main theorems, we develop some estimates for the Jacobian $J(x, f)$ of a mapping $f: \Omega \rightarrow \mathbb{R}^{n}, f=\left(f^{1}, \ldots, f^{n}\right)$. We know that the Jacobian $J(x, f)$ of a mapping $f$ is an $n$-form, specifically, $J(x, f) d x=d f^{1} \wedge \cdots \wedge d f^{n}$, where $d x=d x_{1} \wedge d x_{2} \wedge \cdots \wedge d x_{n}$. For example, let $f=\left(f^{1}, f^{2}\right)$ be a differential mapping in $\mathbb{R}^{2}$. Then,

$$
\begin{aligned}
J(x, f) d x \wedge d y & =\left|\begin{array}{ll}
f_{x}^{1} & f_{y}^{1} \\
f_{x}^{2} & f_{y}^{2}
\end{array}\right| d x \wedge d y \\
& =\left(f_{x}^{1} f_{y}^{2}-f_{y}^{1} f_{x}^{2}\right) d x \wedge d y, \\
d f^{1} \wedge d f^{2} & =\left(f_{x}^{1} d x+f_{y}^{1} d y\right) \wedge\left(f_{x}^{2} d x+f_{y}^{2} d y\right) \\
& =f_{y}^{1} f_{x}^{2} d y \wedge d x+f_{x}^{1} f_{y}^{2} d x \wedge d y \\
& =\left(f_{x}^{1} f_{y}^{2}-f_{y}^{1} f_{x}^{2}\right) d x \wedge d y
\end{aligned}
$$

where we have used the property $d x_{i} \wedge d x_{j}=-d x_{j} \wedge d x_{i}$ if $i \neq j$, and $d x_{i} \wedge d x_{j}=0$ if $i=j$. Clearly, $J(x, f) d x \wedge d y=$ $d f^{1} \wedge d f^{2}$.

Let $f: \Omega \rightarrow \mathbb{R}^{n}, f=\left(f^{1}, \ldots, f^{n}\right)$ be a mapping, whose distributional differential Df $=\left[\partial f^{i} / \partial x_{j}\right]: \Omega \rightarrow \mathrm{GL}(n)$ is 
a locally integrable function on $M$ with values in the space $\mathrm{GL}(n)$ of all $n \times n$-matrices. We use

$$
J(x, f)=\operatorname{det} \operatorname{Df}(x)=\left|\begin{array}{ccccc}
f_{x_{1}}^{1} & f_{x_{2}}^{1} & f_{x_{3}}^{1} & \cdots & f_{x_{n}}^{1} \\
f_{x_{1}}^{2} & f_{x_{2}}^{2} & f_{x_{3}}^{2} & \cdots & f_{x_{n}}^{2} \\
\vdots & \vdots & \vdots & \ddots & \vdots \\
f_{x_{1}}^{n} & f_{x_{2}}^{n} & f_{x_{3}}^{n} & \cdots & f_{x_{n}}^{n}
\end{array}\right|
$$

to denote the Jacobian determinant of $f$. Assume that $u$ is the subdeterminant of Jacobian $J(x, f)$, which is obtained by deleting the $k$ rows and $k$ columns, $k=0,1, \ldots, n-1$; that is,

$$
\begin{aligned}
u & =J\left(x_{j_{1}}, x_{j_{2}}, \ldots, x_{j_{n-k}} ; f^{i_{1}}, f^{i_{2}}, \ldots, f^{i_{n-k}}\right) \\
& =\left|\begin{array}{ccccc}
f_{x_{j_{1}}}^{i_{1}} & f_{x_{j_{2}}}^{i_{1}} & f_{x_{j_{3}}}^{i_{1}} & \cdots & f_{x_{j_{n-k}}}^{i_{1}} \\
f_{x_{j_{1}}}^{i_{2}} & f_{x_{j_{2}}}^{i_{2}} & f_{x_{j_{3}}}^{i_{2}} & \cdots & f_{x_{j_{n-k}}}^{i_{2}} \\
\vdots & \vdots & \vdots & \ddots & \vdots \\
f_{x_{j_{1}}}^{i_{n-k}} & f_{x_{j_{2}}}^{i_{n-k}} & f_{x_{j_{3}}}^{i_{n-k}} & \cdots & f_{x_{j_{n-k}}}^{i_{n-k}}
\end{array}\right|
\end{aligned}
$$

which is an $(n-k) \times(n-k)$ subdeterminant of $J(x, f),\left\{i_{1}, i_{2}, \ldots, i_{n-k}\right\} \quad \subset \quad\{1,2, \ldots, n\}$ and $\left\{j_{1}, j_{2}, \ldots\right.$, $\left.j_{n-k}\right\} \subset\{1,2, \ldots, n\}$. Note that $J\left(x_{j_{1}}, x_{j_{2}}, \ldots, x_{j_{n-k}} ; f^{i_{1}}, f^{i_{2}}, \ldots\right.$, $\left.f^{i_{n-k}}\right) d x_{j_{1}} \wedge d x_{j_{2}} \wedge \cdots \wedge d x_{j_{n-k}}$ is an $(n-k)$-form. Thus, all estimates for differential forms are applicable to the $(n-k)$ form $J\left(x_{j_{1}}, x_{j_{2}}, \ldots, x_{j_{n-k}} ; f^{i_{1}}, f^{i_{2}}, \ldots, f^{i_{n-k}}\right) d x_{j_{1}} \wedge d x_{j_{2}} \wedge \cdots \wedge$ $d x_{j_{n-k}}$. For example, choosing $u=J(x, f) d x$ and applying Theorems 7 and 9 to $u$, respectively, we have the following theorems.

Theorem 11. Let $\varphi$ be a Young function in the class $G(p, q, C)$, $1 \leq p<q<\infty, C \leq 1$. Let $f=\left(f^{1}, \ldots, f^{n}\right): \Omega \rightarrow \mathbb{R}^{n}$ be a mapping such that $J(x, f) d x \in \mathrm{WRH}\left(\wedge^{n}, \Omega\right)$, where $J(x, f)$ is the Jacobian of the mapping $f$ and $\Omega \subset \mathbb{R}^{n}$ is a bounded domain in $\mathbb{R}^{n}$. Assume that $\varphi(|J(x, f)|) \in L_{\text {loc }}^{1}(\Omega, \mu)$. Then, there exists a constant $C$, independent of $J(x, f)$, such that

$$
\begin{gathered}
\int_{B} \varphi\left(\frac{\left|P(J(x, f))-(P(J(x, f)))_{B}\right|}{k} d u\right) \\
\leq C \int_{\sigma B} \varphi\left(\frac{|J(x, f)|}{k}\right) d u
\end{gathered}
$$

for all balls $B \subset \Omega$ and some constant $\sigma>1$.

Theorem 12. Let $\varphi$ be a Young function in the class $G(p, q, C)$, $1 \leq p<q<\infty, C \leq 1$. Let $f=\left(f^{1}, \ldots, f^{n}\right)$ : $\Omega \rightarrow \mathbb{R}^{n}$ be a mapping such that $J(x, f) d x \in \mathrm{WRH}\left(\wedge^{n}, \Omega\right)$, where $J(x, f)$ is the Jacobian of the mapping $f$ and $\Omega \subset \mathbb{R}^{n}$ is a bounded $L^{\varphi}(\mu)$-averaging domain in $\mathbb{R}^{n}$. Assume that
$\varphi(|J(x, f)|) \in L^{1}(\Omega, \mu)$. Then, there exists a constant $C$, independent of $J(x, f)$, such that

$$
\begin{gathered}
\int_{\Omega} \varphi\left(\frac{\left|P(J(x, f))-(P(J(x, f)))_{B_{0}}\right|}{k} d u\right) \\
\quad \leq C \int_{\Omega} \varphi\left(\frac{|J(x, f)|}{k}\right) d u
\end{gathered}
$$

for some ball $B_{0} \subset \Omega$.

\section{References}

[1] R. P. Agarwal, S. Ding, and C. A. Nolder, Inequalities for Differential Forms, Springer, New York, NY, USA, 2009.

[2] H. Bi, "Weighted inequalities for potential operators on differential forms," Journal of Inequalities and Applications, vol. 2010, Article ID 713625, 2010.

[3] H. Bi and Y. Xing, "Poincaré-type inequalities with $L^{p}(\log L)^{\alpha}$ norms for Green's operator," Computers and Mathematics with Applications, vol. 60, no. 10, pp. 2764-2770, 2010.

[4] B. Liu, " $A_{r}^{\lambda}(\Omega)$-weighted imbedding inequalities for Aharmonic tensors," Journal of Mathematical Analysis and Applications, vol. 273, no. 2, pp. 667-676, 2002.

[5] Y. Wang, "Two-weight Poincaré-type inequalities for differential forms in $L^{s}(\mu)$-averaging domains," Applied Mathematics Letters, vol. 20, no. 11, pp. 1161-1166, 2007.

[6] X. Yuming, "Weighted integral inequalities for solutions of the A-harmonic equation," Journal of Mathematical Analysis and Applications, vol. 279, no. 1, pp. 350-363, 2003.

[7] Y. Xing and Y. Wang, "Bmo and Lipschitz norm estimates for composite operators," Potential Analysis, vol. 31, no. 4, pp. 335$344,2009$.

[8] Y. Xing, "A new weight class and Poincaré inequalities with the Radon measures," Journal of Inequalities and Applications, vol. 2012, article 32, 2012.

[9] S. M. Buckley and P. Koskela, "Orlicz-hardy inequalities," Illinois Journal of Mathematics, vol. 48, no. 3, pp. 787-802, 2004.

[10] S. G. Staples, " $L^{p}$-averaging domains and the Poincaré inequality," Annales Academioe Scientiarum Fennicoe Mathematica, vol. 14, pp. 103-127, 1989.

[11] S. Ding and C. A. Nolder, " $L^{s}(\mu)$-averaging domains," Journal of Mathematical Analysis and Applications, vol. 283, no. 1, pp. 85-99, 2003.

[12] S. Ding, " $L^{\varphi}(\mu)$-averaging domains and the quasi-hyperbolic metric," Computers and Mathematics with Applications, vol. 47, no. 10-11, pp. 1611-1618, 2004. 


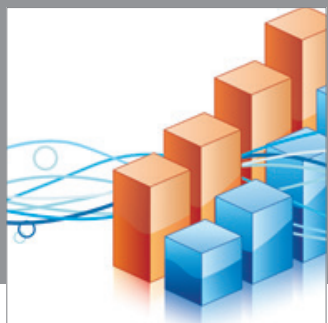

Advances in

Operations Research

mansans

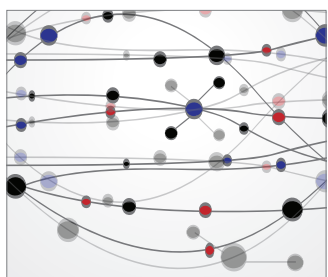

The Scientific World Journal
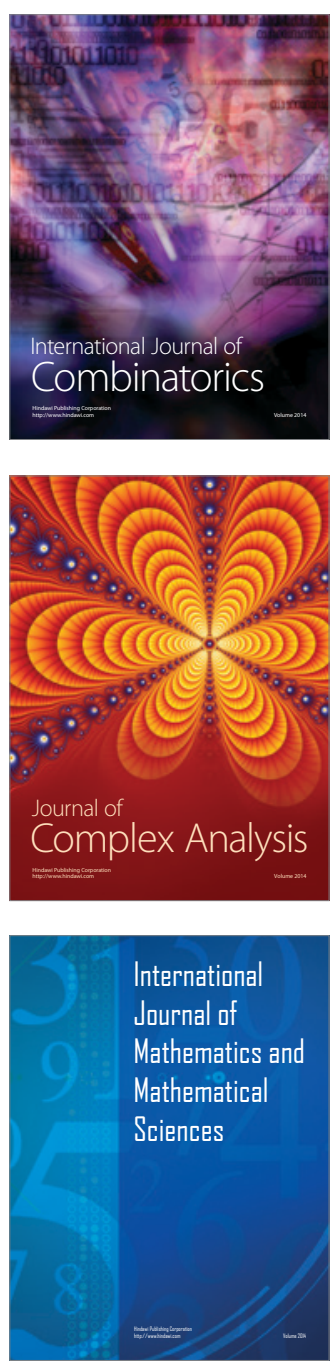
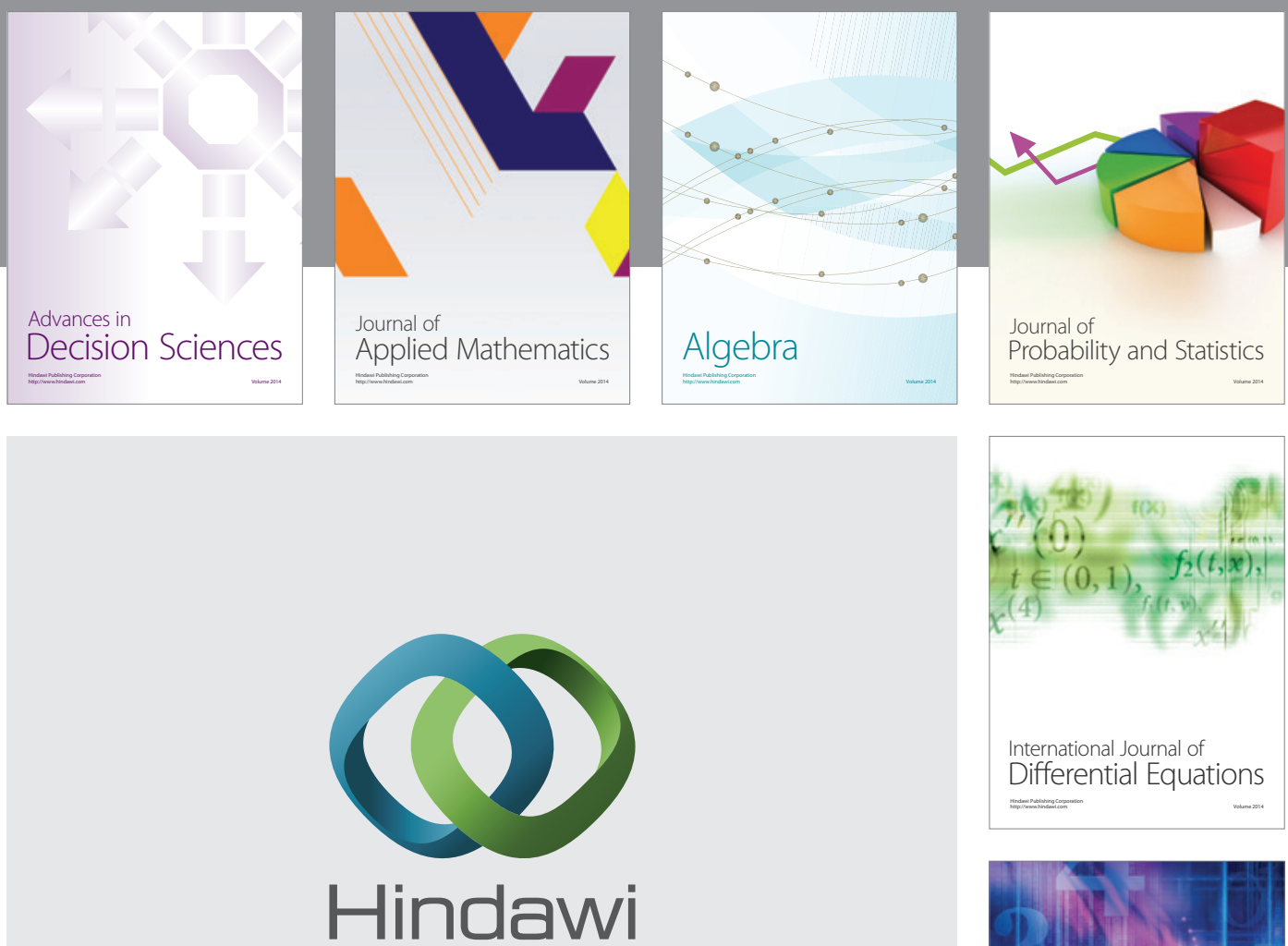

Submit your manuscripts at http://www.hindawi.com
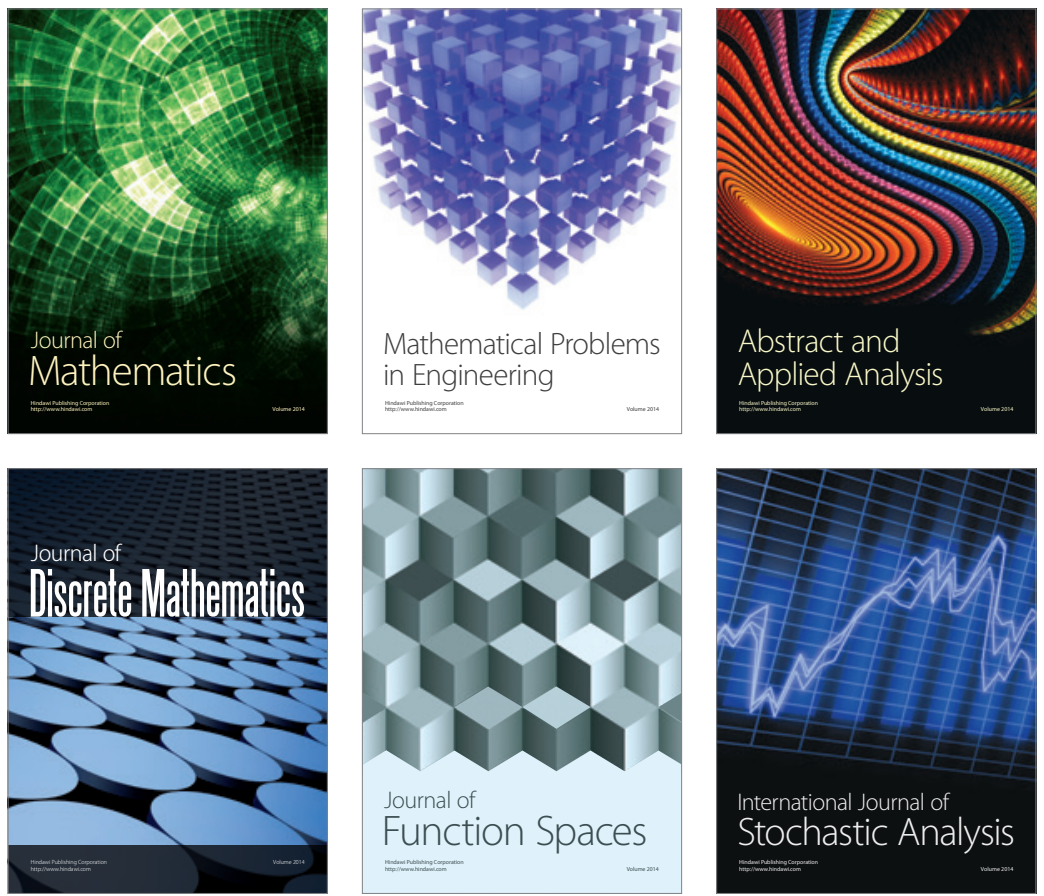

Journal of

Function Spaces

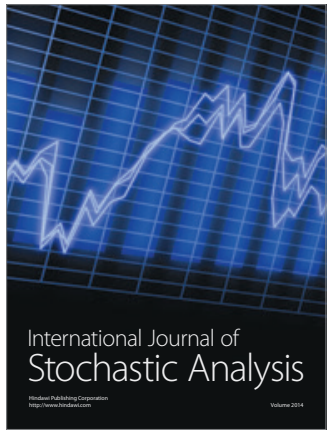

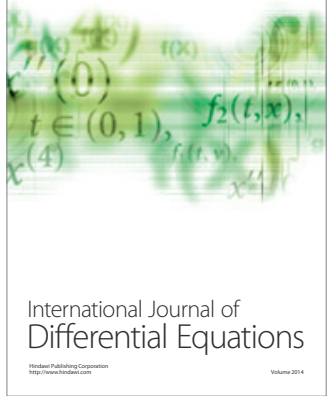
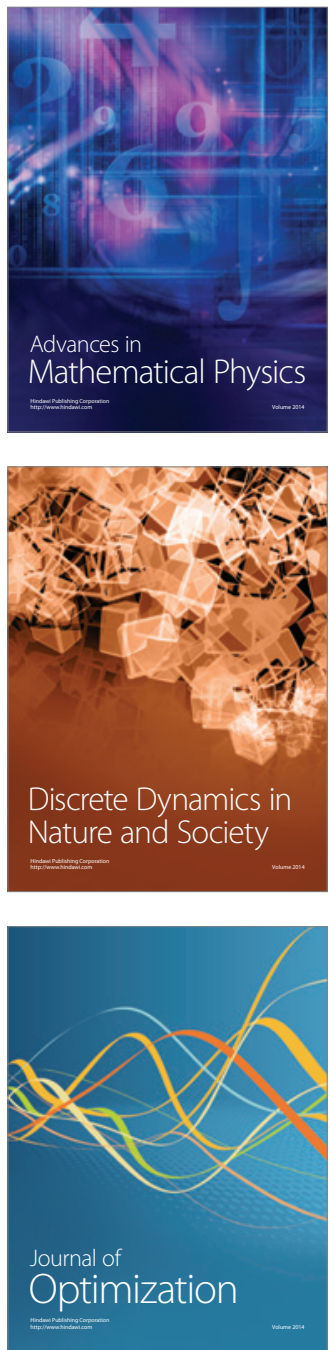